\title{
ON THE COMPARISON THEOREM FOR ÉTALE COHOMOLOGY OF NON-ARCHIMEDEAN ANALYTIC SPACES
}

BY

\author{
VLADIMIR G. BERKOVICH* \\ Department of Theoretical Mathematics \\ The Weizmann Institute of Science \\ P.O.B. 26, 76100 Rehovot, Israel \\ e-mail:vova@wisdom.weizmann.ac.il
}

\begin{abstract}
Let $\varphi: \mathcal{Y} \rightarrow \mathcal{X}$ be a morphism of finite type between schemes of locally finite type over a non-Archimedean field $k$, and let $\mathcal{F}$ be an étale constructible sheaf on $\mathcal{Y}$. In [Ber2] we proved that if the torsion orders of $\mathcal{F}$ are prime to the characteristic of the residue field of $k$ then the canonical homomorphisms $\left(R^{q} \varphi_{*} \mathcal{F}\right)^{\text {an }} \rightarrow R^{q} \varphi_{*}^{\text {an }} \mathcal{F}^{\text {an }}$ are isomorphisms. In this paper we extend the above result to the class of sheaves $\mathcal{F}$ with torsion orders prime to the characteristic of $k$.
\end{abstract}

\section{Introduction}

In [Ber2] (see also [Ber3]), an étale cohomology theory for non-Archimedean analytic spaces has been constructed. In particular, the following two comparison theorems have been proved. Let $\varphi: \mathcal{Y} \rightarrow \mathcal{X}$ be a morphism between schemes of locally finite type over a non-Archimedean field $k$, and let $\mathcal{F}$ be an étale abelian torsion sheaf on $\mathcal{Y}$. The comparison theorem for cohomology with compact support ([Ber2], 7.1.4) states that if the morphism $\varphi$ is compactifiable, then there are canonical isomorphisms

$$
\left(R^{q} \varphi_{!} \mathcal{F}\right)^{\text {an }} \stackrel{\sim}{\rightarrow} R^{q} \varphi_{!}^{\text {an }} \mathcal{F}^{\text {an }}
$$

* Incumbent of the Reiter Family Career Development Chair.

Received May 29, 1994 
The comparison theorem ([Ber2], 7.5.3) states that if $\varphi$ is of finite type and $\mathcal{F}$ is constructible with torsion orders prime to $\operatorname{ch}(\widetilde{k})$, where $\widetilde{k}$ is the residue field of $k$, then there are canonical isomorphisms

$$
\left(R^{q} \varphi_{*} \mathcal{F}\right)^{\text {an }} \stackrel{\sim}{\rightarrow} R^{q} \varphi_{*}^{\text {an }} \mathcal{F}^{\text {an }}
$$

The latter comparison theorem does not say anything on $p$-torsion sheaves when $\operatorname{char}(k)=0$ and $\operatorname{char}(\tilde{k})=p>0$. But the evidence that the isomorphism (*) should be true also in such a situation has been provided by the $p$-adic Riemann existence theorem, proved by W. Lütkebohmert in [Lu2]. It implies straightforwardly that $H^{1}(\mathcal{Y}, \mathbb{Z} / n \mathbb{Z}) \stackrel{\sim}{\rightarrow} H^{1}\left(\mathcal{Y}^{\text {an }}, \mathbb{Z} / n \mathbb{Z}\right)$ for arbitrary $n$ prime to $\operatorname{char}(k)$.

The main purpose of this paper is to prove that the isomorphism $(*)$ really takes place without any restriction on the torsion orders of $\mathcal{F}$ in the case when $k$ is of characteristic zero. The proof is given in $\S 3$ and follows the proof of the comparison theorem of M. Artin and A. Grothendieck ([SGA4], Exp. XVI, 4.1). Using Hironaka's theorem on resolution of singularities, the weak base change theorem ([Ber2], 5.3.1) and the comparison theorem for cohomology with compact support, the situation is reduced to the case when $\mathcal{X}$ is smooth, $\varphi$ is an open immersion, and $\mathcal{F}=\Lambda_{\mathcal{Y}}$, where $\Lambda=\mathbb{Z} / n \mathbb{Z}$. In this case, the isomorphism (*) for $q=0$ follows from the $p$-adic Riemann extension theorem, proved by W. Lütkebohmert in [Lu1], and the verification of (*) for $q \geq 1$ is reduced to the case when $\mathcal{Z}:=\mathcal{X} \backslash \mathcal{Y}$ is also smooth. If $i$ denotes the closed immersion $\mathcal{Z} \rightarrow \mathcal{X}$, then (*) is equivalent to the fact that the canonical homomorphism

$$
\left(R^{q} i \Lambda_{\mathcal{X}}\right)^{\text {an }} \rightarrow R^{q} i^{\text {an } !} \Lambda_{\mathcal{X} \otimes n}
$$

is an isomorphism. The latter is deduced from the cohomological purity theorem proved in $\S 2$. Using a result of $\mathrm{W}$. Lütkebohmert from [Lu2], we prove that the affine space is universally acyclic, and deduce from this that if $(Y, X)$ is a smooth $S$-pair of codimension $c$, then $R^{q} i \Lambda_{X}=0$ for $q \neq 2 c$ and $R^{2 c} i ! \Lambda_{X}$ is locally isomorphic to $\Lambda_{Y}$. (In particular, the both sheaves in (?) are locally isomorphic.) Furthermore, we construct an isomorphism $R^{2 c} i^{!} \Lambda_{X}(c) \stackrel{\sim}{\rightarrow} \Lambda_{Y}$ and establish its properties which guarantee that (?) is an isomorphism. For this we use the Verdier duality theorem, proved in $\S 1$, and the trace mapping $R^{2 d} \varphi_{!} \Lambda_{Y}(d) \rightarrow \Lambda_{X}$ constructed in [Ber2], $\$ 7.2$, for any separated smooth morphism $\varphi: Y \rightarrow X$ of pure 
dimension $d$ and any $n$ prime to $\operatorname{char}(k)$. (In [Ber2], the trace mapping was used only for $n$ prime to $\operatorname{char}(\tilde{k})$.)

Throughout the paper we fix a non-Archimedean field $k$, a positive integer $n$, and we set $\Lambda=\mathbb{Z} / n \mathbb{Z}$. (As in [Ber1]-[Ber3], the valuation on $k$ is not assumed to be nontrivial.)

\section{Verdier Duality}

1.1 Theorem: Let $\varphi: Y \rightarrow X$ be a Hausdorff morphism of finite dimension between $k$-analytic spaces. Then there is an exact functor

$$
R \varphi^{!}: D^{+}(X, \Lambda) \rightarrow D^{+}(Y, \Lambda)
$$

and, for any $G^{\cdot} \in D^{-}(Y, \Lambda)$ and $F^{\cdot} \in D^{+}(X, \Lambda)$, a functorial isomorphism

$$
R \varphi_{*}\left(\underline{\mathcal{H} o m}\left(G^{*}, R \varphi^{!} F^{*}\right)\right) \stackrel{\sim}{\rightarrow} \underline{\mathcal{H} o m}\left(R \varphi_{!} G^{*}, F^{\prime}\right) .
$$

It is clear that Theorem 1.1 will be proved if we construct the functor $R \varphi !$ and prove the following

1.2 COROllary: There is a functorial isomorphism

$$
\underline{\operatorname{Hom}}\left(G^{\prime}, R \varphi^{\prime} F^{\prime}\right) \stackrel{\sim}{\operatorname{Hom}}\left(R \varphi ! G^{*}, F^{\prime}\right) .
$$

Proof: Let $d=\operatorname{dim}(\varphi)$. We say that a sheaf $L \in \mathbf{S}(Y, \Lambda)$ is strongly $\varphi$ !-acyclic if, for any separated étale morphism $g: V \rightarrow Y$, the sheaf $L_{V / Y}=g !\left(\left.L\right|_{V}\right)$ is $\varphi_{!-}$ acyclic.

1.3 Lemma: If a sheaf $L \in \mathbf{S}(Y, \Lambda)$ is flat strongly $\varphi_{!}$-acyclic, then for any $G \in \mathbf{S}(Y, \Lambda)$ the sheaf $L \otimes G$ is $\varphi$ !-acyclic.

Proof: Take a resolution of $G$

$$
\ldots \rightarrow G_{1} \rightarrow G_{0} \rightarrow G \rightarrow 0
$$

whose members are of the form $\bigoplus_{i} \Lambda_{V_{i} / Y}$, where $V_{i} \rightarrow Y$ are separated étale morphisms. Tensoring it with $L$, we get an exact sequence

$$
\cdots \stackrel{d_{2}}{\rightarrow} L \otimes G_{1} \stackrel{d_{1}}{\rightarrow} L \otimes G_{0} \stackrel{d_{0}}{\rightarrow} L \otimes G \rightarrow 0
$$


whose members are of the form $L \otimes\left(\bigoplus_{i} \Lambda_{V_{i} / Y}\right)=\bigoplus_{i} L_{V_{i} / Y}$. Since the functor $\varphi$ ! commutes with direct sums, all the sheaves $L \otimes G_{m}$ are $\varphi_{\text {! }}$-acyclic. It follows that for $q \geq 1$ one has

$$
R^{q} \varphi_{!}(L \otimes G) \stackrel{\sim}{\rightarrow} R^{q+2 d} \varphi !\left(\operatorname{Ker} d_{2 d-1}\right)=0
$$

because $R^{q} \varphi_{1}=0$ for $q>2 d$, by [Ber2], 5.3.8.

For a flat strongly $\varphi$ !-acyclic sheaf $L \in \mathbf{S}(Y, \Lambda)$, we denote by $\varphi_{!}^{L}$ the following functor

$$
\mathbf{S}(Y, \Lambda) \rightarrow \mathbf{S}(X, \Lambda): G \mapsto \varphi !(L \otimes G) .
$$

1.4 LEMMA: The functor $\varphi_{!}^{L}$ is exact and has a right adjoint functor $\varphi_{L}^{!}: \mathbf{S}(X, \Lambda) \rightarrow \mathbf{S}(Y, \Lambda)$. The functor $\varphi_{L}^{!}$takes injectives to injectives.

Proof: Let $0 \rightarrow G^{\prime} \rightarrow G \rightarrow G^{\prime \prime} \rightarrow 0$ be an exact sequence of sheaves on $Y$. Since $L$ is flat, the sequence $0 \rightarrow L \otimes G^{\prime} \rightarrow L \otimes G \rightarrow L \otimes G^{\prime \prime} \rightarrow 0$ is also exact. By Lemma $1.3, R^{1} \varphi_{!}\left(L \otimes G^{\prime}\right)=0$, and therefore the sequence $0 \rightarrow \varphi_{!}^{L}\left(G^{\prime}\right) \rightarrow$ $\varphi_{!}^{L}(G) \rightarrow \varphi_{!}^{L}\left(G^{\prime \prime}\right) \rightarrow 0$ is exact. Furthermore, we claim that for any $F \in \mathbf{S}(X, \Lambda)$ the contravariant functor

$$
\mathbf{S}(Y, \Lambda) \rightarrow \mathcal{A} b: G \mapsto \operatorname{Hom}\left(\varphi_{!}^{L}(G), F\right)
$$

is representable. Indeed, for this it suffices to verify that this functor takes inductive limits to projective limits (see [SGA4], Exp. XVIII, 3.1.3). But this follows from the facts that the functor $\varphi_{!}^{L}$ is exact and that the tensor product

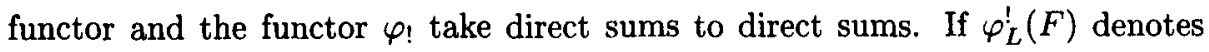
a sheaf which represents the functor considered, then the correspondence $F \mapsto$ $\varphi_{L}^{!}(F)$ is a functor right adjoint to $\varphi_{!}^{L}$. The last statement of the lemma follows from the fact that the functor $\varphi_{!}^{L}$ is exact.

1.5 Proposition: Any flat sheaf $G \in \mathbf{S}(Y, \Lambda)$ has a resolution

$$
0 \rightarrow G \rightarrow L^{0} \rightarrow L^{1} \rightarrow \cdots \rightarrow L^{2 d} \rightarrow 0
$$

in which all $L^{i}$ are flat strongly $\varphi_{!}$-acyclic sheaves.

Proof: 1. Recall the construction of the Godement resolution from [SGA4], Exp. XVII, $\S 4.2$, adopted to our situation. Suppose we are given a set $I$, a surjective map $\sigma: I \rightarrow Y$ and, for each $i \in I$, an algebraically closed non-Archimedean 
field $K_{i}$ over $\mathcal{H}(\sigma(i))$. These data define a morphism of analytic spaces over $k$, $\nu: \mathcal{Y} \rightarrow Y$, where $\mathcal{Y}$ is the disjoint union of $\mathcal{M}\left(K_{i}\right)$ over all $i \in I$. For a sheaf $G \in \mathbf{S}(Y, \Lambda)$, let $\mathcal{C}(G)$ denote the right resolution of $G$ constructed as follows:

(a) $\mathcal{C}^{0}(G)=\nu_{*} \nu^{*}(G)$, and $\varepsilon=d^{-1}: G \rightarrow \mathcal{C}^{0}(G)$ is the adjunction morphism;

(b) if $m \geq 0$, then $\mathcal{C}^{m+1}(G)=\mathcal{C}^{0}\left(\operatorname{Coker} d^{m-1}\right)$, and $d^{m}$ is the composition $d^{m}: \mathcal{C}^{m}(G) \rightarrow$ Coker $d^{m-1} \rightarrow \mathcal{C}^{0}\left(\right.$ Coker $\left.d^{m-1}\right)$.

By loc. cit., 4.2.3, one has:

(i) $\mathcal{C}^{m}(G)$ is a flabby sheaf;

(ii) the functor $G \mapsto \mathcal{C}^{m}(G)$ is exact;

(iii) the fibre of the complex $\mathcal{C}(G)$ at a point $y \in Y$ is a canonically split resolution of $G_{y}$.

1.6 LEMMA: The sheaves $\mathcal{C}^{m}(G)$ are strongly $\varphi_{\text {!-acyclic. }}$

Proof: It suffices to verify the statement for $m=0$. We have to show that, for any separated étale morphism $g: V \rightarrow Y, R^{q}(\varphi g)_{!}\left(\left.\mathcal{C}^{0}(G)\right|_{V}\right)=0, q \geq 1$. Replacing the set $I$ by another one, we may replace $Y$ by $V$, and so we have to show that $R^{q} \varphi_{1}\left(\mathcal{C}^{0}(G)\right)=0, q \geq 1$. Since the statement is local with respect to the étale topology of $X$ and the sheaf $R^{q} \varphi_{!}\left(\mathcal{C}^{0}(G)\right)$ is associated with the presheaf $(U \stackrel{f}{\rightarrow} X) \mapsto H_{\mathcal{C}_{\varphi}(f)}^{q}\left(Y \times_{X} U, \mathcal{C}^{0}(G)\right)$, where $\mathcal{C}_{\varphi}$ is the $\varphi$-family of supports defined in [Ber2], 5.1.3, it suffices to show that in the case of paracompact $X$ one has $H_{\Phi}^{q}\left(Y, \mathcal{C}^{0}(G)\right)=0$ for all $q \geq 1$, where $\Phi=\mathcal{C}_{\varphi}(\mathrm{Id})$. For this we use the spectral sequence $E_{2}^{p, q}=H_{\Phi}^{p}\left(|Y|, R^{q} \pi_{*}\left(\mathcal{C}^{0}(G)\right)\right) \Longrightarrow H_{\Phi}^{p+q}\left(Y, \mathcal{C}^{0}(G)\right)$, where $\pi$ is the morphism of sites $Y_{\dot{e} t} \rightarrow|Y|$. The sheaf $\mathcal{C}^{0}(G)$ is flabby, and therefore $R^{q} \pi_{*}\left(\mathcal{C}^{0}(G)\right)=0$ for $q \geq 1$, by [Ber2], 4.2.5. Furthermore, from the construction of $\mathcal{C}^{0}(G)$ it follows that the sheaf $\pi_{*}\left(\mathcal{C}^{0}(G)\right)$ is flasque in the sense of [God]. Since the family of supports $\Phi$ is paracompactifying, it follows that the latter sheaf is $\Phi$-soft, and therefore $H_{\Phi}^{p}\left(|Y|, \pi_{*}\left(\mathcal{C}^{0}(G)\right)\right)=0$ for all $p \geq 1$.

2. Suppose now that $G$ is flat. We set $L^{m}=\mathcal{C}^{m}(G)$ for $0 \leq m \leq 2 d-1$, and $L^{2 d}=\operatorname{Ker}\left(d^{2 d}\right)$. From 1(iii) it follows that all the sheaves $L^{0}, \ldots, L^{2 d}$ are flat. Let $V \rightarrow Y$ be a separated étale morphism. By Lemma 1.6, the sheaves $L^{0}, \ldots, L^{2 d-1}$ are strongly $\varphi_{!}$-acyclic, and therefore

$$
R^{q} \varphi_{!}\left(L_{V / Y}^{2 d}\right) \stackrel{\sim}{\rightarrow} R^{q+2 d} \varphi_{!}\left(G_{V / Y}\right)=0
$$

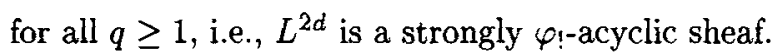




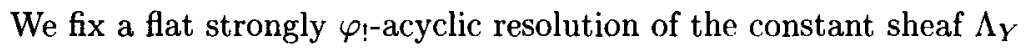

$$
0 \rightarrow \Lambda_{Y} \rightarrow L^{0} \rightarrow L^{1} \rightarrow \ldots \rightarrow L^{2 d} \rightarrow 0 .
$$

For a complex $G^{\cdot} \in C^{-}(Y, \Lambda)$, let $\varphi_{!}^{L}\left(G^{\cdot}\right)$ denote the complex $\varphi_{!}\left(L \otimes G^{\cdot}\right)$. Furthermore, for a complex $F^{\cdot} \in C^{+}(X, \Lambda)$, let $\varphi_{L^{\prime}}^{!}\left(F^{\cdot}\right)$ denote the simple complex associated with the double complex $K^{p, q}=\varphi_{L^{-p}}^{!}\left(F^{q}\right)$. It follows that there is a functorial isomorphism

$$
\operatorname{Hom}^{\cdot}\left(G^{*}, \varphi_{L^{\prime}}^{\prime}\left(F^{\prime}\right)\right) \stackrel{\sim}{\rightarrow} \operatorname{Hom}\left(\varphi_{!}^{L}\left(G^{*}\right), F^{*}\right) .
$$

We now define the functor $R \varphi^{\prime}: D^{+}(X, \Lambda) \rightarrow D^{+}(Y, \Lambda)$ as follows. Let $F \rightarrow I$ be an injective resolution of a complex $F^{\cdot} \in C^{+}(X, \Lambda)$. We set

$$
R \varphi^{!} F^{\cdot}=\varphi_{L}^{!} \cdot\left(I^{\prime}\right)
$$

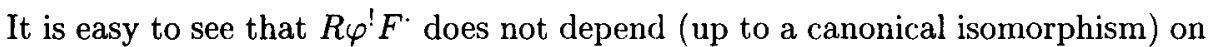
the choice of the resolution $I^{\cdot}$ and that, for $G \in D^{-}(Y, \Lambda)$ and $F^{\cdot} \in D^{+}(X, \Lambda)$, there is a functorial isomorphism $\underline{\operatorname{Hom}}\left(G^{\prime}, R \varphi^{\prime} F^{\prime}\right) \stackrel{\sim}{\rightarrow} \underline{\operatorname{Hom}}\left(R \varphi_{!} G^{\prime}, F^{*}\right)$. Theorem 1.1 is proved.

1.7. Remarks: (i) From the construction of $R \varphi^{\prime}$ it follows that if the cohomology sheaves of a complex $F^{\cdot} \in D^{+}(X, \Lambda)$ are trivial at dimensions $<q$, then the cohomology sheaves of the complex $R \varphi^{\prime} F^{\cdot} \in D^{+}(Y, \Lambda)$ are trivial at dimensions $<q-2 d$.

(ii) If $\psi: Z \rightarrow Y$ is a similar morphism, then the canonical isomorphism of func-

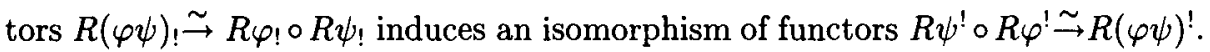

(iii) Suppose that $d=0$. Then $R \varphi$ ! is actually the right derived functor of a left exact functor $\varphi^{!}: \mathbf{S}(X, \Lambda) \rightarrow \mathbf{S}(Y, \Lambda)$ defined as follows

$$
\Gamma\left(V, \varphi^{!}(F)\right)=\operatorname{Hom}\left(\varphi_{!}\left(\Lambda_{V / Y}\right), F\right) .
$$

Moreover, $\varphi^{!}$is right adjoint to $\varphi_{1}$. If $\varphi$ is étale, then $\varphi^{!}=\varphi^{*}$. If $\varphi$ is a quasiimmersion ([Ber2], §4.3) such that $\varphi(Y)$ is closed in $X$, then $\varphi^{!}$is the functor of sections with supports in $\varphi(Y)$ (defined in [Ber2], §5.1.1), and the sheaves $R^{q} \varphi^{!}(F)$ were denoted in [Ber2] by $\mathcal{H}_{Y}^{q}(X, F)$.

The complex $R \varphi \Lambda_{X}$ is said to be the dualizing complex of the morphism $\varphi$ and is denoted by $T_{Y / X}$ (if $X=\mathcal{M}(k)$, it is denoted by $T_{Y}$ ). By Remark 1.7(i), $H^{q}\left(T_{Y / X}\right)=0$ for $q<-2 d$. 
Let $\varphi: Y \rightarrow X$ be a separated smooth morphism of pure dimension $d$, and assume that $n$ is prime to $\operatorname{char}(k)$. In [Ber2], $\S 7.2$, we constructed a canonical homomorphism of sheaves (the trace mapping)

$$
\operatorname{Tr}_{\varphi}: R^{2 d} \varphi_{!} \Lambda_{Y}(d) \rightarrow \Lambda_{X} .
$$

Recall also that if the fibres of $\varphi$ are non-empty, then $\operatorname{Tr}_{\varphi}$ is an epimorphism and if, in addition, the geometric fibres of $\varphi$ are connected and $n$ is prime to $\operatorname{char}(\widetilde{k})$, then $\operatorname{Tr}_{\varphi}$ is an isomorphism. By Theorem 1.1, the trace mapping induces a morphism of complexes $t_{\varphi}: \Lambda_{Y} \rightarrow T_{Y / X}(-d)[-2 d]$ or, equivalently, a homomorphism of sheaves $c_{\varphi}=H^{0}\left(t_{\varphi}\right): \Lambda_{Y} \rightarrow H^{-2 d}\left(T_{Y / X}^{\cdot}(-d)\right)$. The image of 1 under $c_{\varphi}$ is called the fundamental class of $\varphi$, and so $t_{\varphi}$ and $c_{\varphi}$ will be called the fundamental class mappings. By Poincaré Duality Theorem ([Ber2], 7.3.1), if $n$ is prime to $\operatorname{char}(\widetilde{k})$, then $t_{\varphi}$ (and therefore $c_{\varphi}$ ) is an isomorphism. We claim that in the general case (when $n$ is prime only to $\operatorname{char}(k)$ ) the homomorphism $c_{\varphi}$ is injective. Indeed, to verify this, it suffices to assume that $n$ is a prime integer. The set of points over which the homomorphism $c_{\varphi}$ is not injective is open, and so shrinking $Y$ we may assume that the morphism $t_{\varphi}$ is zero. Furthermore, since a smooth morphism is an open map ([Ber2], 3.7.4), we can shrink $X$ and assume that $\varphi$ is surjective. In this case the vanishing of $t_{\varphi}$ contradicts to the surjectivity of the trace mapping $\operatorname{Tr}_{\varphi}$. The following proposition lists properties of the fundamental class mappings which follow straightforwardly from the properties of the trace mappings established in [Ber2], $\S 7.2$.

1.8 Proposition: The fundamental class mappings $t_{\varphi}$ have the following properties and are uniquely determined by them:

(a) $t_{\varphi}$ are compatible with base change, i.e., given a cartesian diagram

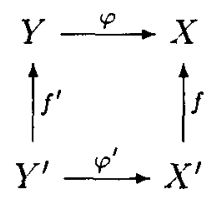

the following diagram is commutative

$$
f^{\prime *}\left(T_{Y / X}\right)(-d)[-2 d] \longrightarrow T_{Y^{\prime} / X^{\prime}}(-d)[-2 d]
$$


(b) $t_{\varphi}$ are compatible with composition, i.e., given a separated smooth morphism $\psi: Z \rightarrow Y$ of pure dimension $e$, the following diagram is commutative

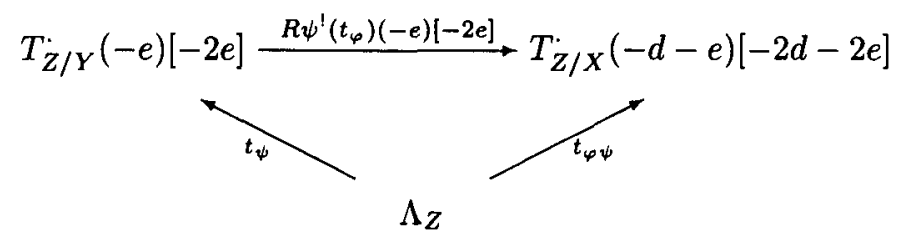

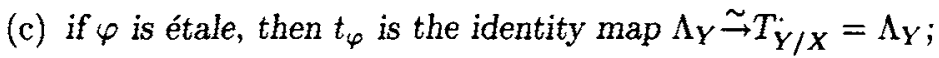

(d) if $\varphi: \mathcal{Y} \rightarrow \mathcal{X}$ is a separated smooth morphism of pure dimension $d$ between schemes of locally finite type over $\operatorname{Spec}(\mathcal{A})$, where $\mathcal{A}$ is a $k$-affinoid algebra, then the following diagram is commutative

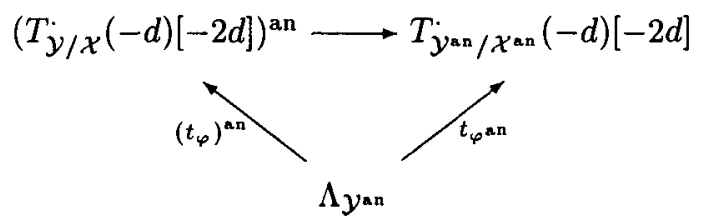

(Recall that, by Poincaré Duality for schemes, $t_{\varphi}$ is an isomorphism.)

\section{Cohomological Purity Theorem}

In this section the integer $n$ is assumed to be prime to char $(k)$.

Let $S$ be a $k$-analytic space. Recall ([Ber2], §7.4) that a smooth $S$-pair $(Y, X)$ is a commutative diagram of morphisms of $k$-analytic spaces

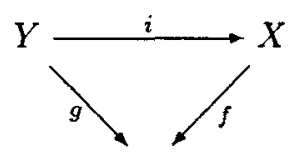

$S$

where $f$ and $g$ are smooth, and $i$ is a closed immersion. The codimension of $(Y, X)$ at a point $y \in Y$ is the codimension at $y$ of the fibre $Y_{s}$ in $X_{s}$, where $s=g(y)$. Given a smooth $S$-pair $(Y, X)$, we denote by $j$ the open immersion $U:=X \backslash Y \hookrightarrow S$ and by $h$ the induced morphism $U \rightarrow S$. Recall also ([Ber2], $\S 1.5)$ that a $k$-analytic space is said to be good if each point of it has an affinoid neighborhood. 
2.1 ThEOREM: Let $(Y, X)$ be a smooth $S$-pair of codimension $c$, and assume that $S$ is good. Then

(i) for any abelian sheaf $F$ on $X$ which is locally isomorphic (in the étale topology) to a sheaf of the form $f^{*} G$, where $G$ is an étale $\Lambda_{S}$-module, one has $R^{q} i^{!} F=0$ for $q \neq 2 c$ and $i^{*} F \otimes R^{2 c} i^{!} \Lambda_{X} \stackrel{\sim}{\rightarrow} R^{2 c} i^{!} F$.

(ii) there is a canonical isomorphism $R^{2 c} i^{!} \Lambda_{X}(c) \stackrel{\sim}{\rightarrow} \Lambda_{Y}$ such that if $g$ is of pure dimension $e$, then the following diagram is commutative

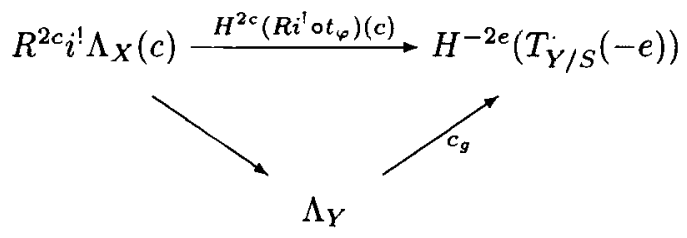

2.2 Lemma (Universal acyclicity of the affine space): Let $X$ be a $k$-analytic space, and let $\varphi$ be the canonical projection $\varphi: X \times \mathbb{A}^{d} \rightarrow X$. Then for any étale $\Lambda_{X}$-module $F$ one has $F \stackrel{\sim}{\rightarrow} \varphi_{*} \varphi^{*} F$ and $R^{q} \varphi_{*}\left(\varphi^{*} F\right)=0$ for all $q \geq 1$.

Proof: We may assume that $d=1$. The isomorphism $F \sim \varphi_{*} \varphi^{*} F$ follows from [Ber2], 7.3.2. Since $R^{q} \varphi_{*}\left(\varphi^{*} F\right)$ is associated with the presheaf $(U \rightarrow X) \mapsto$ $H^{q}\left(\mathbb{A}_{U}^{1}, \varphi^{*} F\right)$, where $\mathbb{A}_{U}^{1}=U \times \mathbb{A}^{1}$, it suffices to show that if $X$ is paracompact then $H^{q}(X, F) \stackrel{\sim}{\rightarrow} H^{q}\left(\mathbb{A}_{X}^{1}, \varphi^{*} F\right)$.

Take a number $r>1$ and denote by $\varphi_{m}$ the canonical projection $Y_{m}:=X \times$ $E\left(0, r^{m}\right) \rightarrow X$, where $E\left(0, r^{m}\right)$ is the closed disc in $\mathbb{A}^{1}$ of radius $r^{m}$ with center at zero. The paracompact $k$-analytic space $\mathbb{A}_{X}^{1}$ is a union of the increasing sequence of the closed $k$-analytic domains $Y_{m}$. From [Ber2], 5.3.8 and 6.1.3, it follows that $R^{q} \varphi_{m_{*}}\left(\varphi_{m}^{*} F\right)=0$ for $q \geq 2$. If $n$ is prime to $\operatorname{char}(\widetilde{k})$, then $R^{1} \varphi_{m_{*}}\left(\varphi_{m}^{*} F\right)=0$, and therefore $H^{q}(X, F) \stackrel{\sim}{\rightarrow} H^{q}\left(Y_{m}, \varphi_{m}^{*} F\right)$ and $H^{q}(X, F) \stackrel{\sim}{\rightarrow} H^{q}\left(\mathbb{A}_{X}^{1}, \varphi^{*} F\right)$ for all $q \geq 1$, by [Ber2], 6.3.12.

Assume now that $\operatorname{char}(k)=0, p:=\operatorname{char}(\tilde{k})>0$ and $n=p^{d}$ for some $d \geq 1$. By Lütkebohmert's Theorem ( $[\mathrm{Lu} 2], 2.1$ ), there exists a constant $0<\varepsilon<1$ depending only on $p$ and $d$ such that for any algebraically closed non-Archimedean field $K$ with $\operatorname{char}(K)=0$ and $\operatorname{char}(\widetilde{K})=p$ and for any $R>0$ the following holds. Any finite étale covering of the closed disc $E(0, R)$ over $K$ of degree at most $p^{d}$ splits over $E(0, \varepsilon R)$. The latter implies that for any $\Lambda$-module $M$ the restriction homomorphism $H^{1}(E(0, R), M) \rightarrow H^{1}(E(0, \varepsilon R), M)$ is zero. If we now choose the number $r$ so that $\varepsilon r>1$, then [Ber2], 5.3.1, implies that the canonical homomorphism $R^{1} \varphi_{m+1}\left(\varphi_{m+1}^{*} F\right) \rightarrow R^{1} \varphi_{m_{*}}\left(\varphi_{m}^{*} F\right)$ is zero. Using the spectral 
sequence $E_{2}^{p, q}=H^{p}\left(X, R^{q} \varphi_{m_{*}}\left(\varphi_{m}^{*} F\right)\right) \Longrightarrow H^{p+q}\left(Y_{m}, \varphi_{m}^{*} F\right)$ and the fact that $R^{q} \varphi_{m *}\left(\varphi_{m}^{*} F\right)=0$ for $q \geq 2$, we get that the image of $H^{q}\left(Y_{m+1}, \varphi_{m+1}^{*} F\right)$ in $H^{q}\left(Y_{m}, \varphi_{m}^{*} F\right)$ coincides with the image of $H^{q}(X, F)$. By [Ber2], 6.3.12, one has $H^{q}(X, F) \stackrel{\sim}{\rightarrow} H^{q}\left(\mathbb{A}_{X}^{1}, \varphi^{*} F\right)$ for all $q \geq 1$. The lemma is proved.

Proof of Theorem 2.1: To construct the isomorphism (ii), it suffices to show that the canonical homomorphism $R^{2 c} i^{\prime} \Lambda_{X}(c) \rightarrow H^{-2 e}\left(T_{Y / S}^{\cdot}(-e)\right)$ identifies the first sheaf with the image of $\Lambda_{Y}$ under the injective homomorphism $c_{g}$. Furthermore, since the formation of $R i^{!}$commutes with any étale base change, we can apply Proposition 3.5.9 from [Ber2] (where the assumption that $S$ is good is used) and assume that $(Y, X)$ is the pair $\left(\mathbb{A}_{S}^{d-c}, \mathbb{A}_{S}^{d}\right)$ and $F$ is of the form $f^{*} G$.

STEP 1: (i) is true and the sheaf $R^{2 c} i \Lambda_{X}(c)$ is isomorphic to $\Lambda_{Y}$ (here $S$ is not necessarily good).

Consider first the case $c=1$. Using Proposition 1.8(b), we may replace $S$ by $\mathbb{A}_{S}^{d-1}$ and assume that $Y$ is the zero section in the affine line $X=\mathbb{A}_{S}^{1}$. After that we may assume that $X=\mathbb{P}_{S}^{1}$ and $Y$ is the section at infinity. Consider the spectral sequence

$$
E_{2}^{p, q}=R^{p} f_{*}\left(R^{q} j_{*}\left(h^{*} G\right)\right) \Longrightarrow R^{p+q} h_{*}\left(h^{*} G\right)
$$

First of all, we claim that $f^{*} G \stackrel{\sim}{\rightarrow} j_{*}\left(h^{*} G\right)$. Indeed, let $F^{\prime}$ be the sheaf defined by the exact sequence

$$
0 \rightarrow f^{*} G \rightarrow j_{*}\left(h^{*} G\right) \rightarrow F^{\prime} \rightarrow 0 .
$$

By [Ber2], 5.3.1, $R^{1} f_{*}\left(f^{*} G\right)=0$ and, by [Ber2], 7.3.2, $G \stackrel{\sim}{\rightarrow} f_{*}\left(f^{*} G\right) \stackrel{\sim}{\rightarrow} h_{*}\left(h^{*} G\right)$. It follows that $f_{*} F^{\prime}=0$, and therefore $F^{\prime}=0$. Thus, $E_{2}^{p, 0}=R^{p} f_{*}\left(f^{*} G\right)=0$ for $p \neq 0,2$, and, by [Ber2], 5.3.9, $G(-1) \stackrel{\sim}{\rightarrow} G \otimes R^{2} f_{*} \Lambda_{X} \stackrel{\sim}{\rightarrow} E_{2}^{2,0}=R^{2} f_{*}\left(f^{*} G\right)$.

Furthermore, since the supports of the sheaves $R^{q} j_{*}\left(h^{*} G\right)$ for $q \geq 1$ are contained in $Y$ and $g$ is an isomorphism, then $E_{2}^{p, q}=0$ for $p \geq 1$ and $q \geq 1$ and $E_{2}^{0, q}=g_{*} i^{*}\left(R^{q} j_{*}\left(h^{*} G\right)\right)$ for $q \geq 1$. By Lemma $2.2, R^{q} h_{*}\left(h^{*} G\right)=0$ for $q \geq 1$, and therefore the spectral sequence implies that $E_{2}^{0, q}=0$ for $q \geq 2$ and $E_{2}^{0,1} \simeq E_{2}^{2,0}$. It follows that $R^{q} j_{*}\left(h^{*} G\right)=0$ for $q \geq 2$ and $R^{1} j_{*}\left(h^{*} G\right) \stackrel{\sim}{\rightarrow} i_{*}\left(g^{*} G\right)(-1)$. Step 1 for $c=1$ now follows from [Ber2], 5.2.7.

The case $c>1$ is verified by induction. Let $c=a+b$, where $a, b>0$. We set $Z=\mathbb{A}_{S}^{d-b}$ and denote by $\mu$ (resp. $\nu$ ) the closed immersion $Y \rightarrow Z$ (resp. $Z \rightarrow X$ ). Consider the spectral sequence

$$
E_{2}^{p, q}=R^{p} \mu^{!}\left(R^{q} \nu^{!} f^{*} G\right) \Longrightarrow R^{p+q} i^{!}\left(f^{*} G\right)
$$


By induction, $R^{q} \nu^{!} f^{*} G=0$ for $q \neq 2 b$ and $R^{2 b} \nu^{!} f^{*} G \stackrel{\sim}{\rightarrow} \nu^{*} f^{*} G(-b)$. Similarly, $E_{2}^{p, 2 b}=0$ for $p \neq 2 b$ and

$$
g^{*} G(-c)=g^{*} G(-b) \otimes \Lambda_{Y}(-a) \stackrel{\sim}{\rightarrow} R^{2 a} \mu^{!}\left(R^{2 b} \nu^{!} f^{*} G\right)=E_{2}^{2 a, 2 b} .
$$

Step 1 follows.

STEP 2: (ii) is true.

Since $S$ is good, we can shrink it and assume that $S=\mathcal{M}(\mathcal{A})$ is $k$-affinoid. Then $(Y, X)$ is the analytification of the smooth $\mathcal{S}$-pair $(\mathcal{Y}, \mathcal{X})=\left(A_{\mathcal{S}}^{d-c}, A_{\mathcal{S}}^{d}\right)$, where $\mathcal{S}=\operatorname{Spec}(\mathcal{A})$. By Poincaré Duality for schemes, the fundamental class mapping $\Lambda_{\mathcal{Y}} \rightarrow T_{\mathcal{Y} / \mathcal{S}}(-e)[-2 e], e=d-c$, is an isomorphism. Using Proposition 1.8(d), we get that the image of $R^{2 c} i^{!} \Lambda_{X}(c)$ in $H^{-2 e}\left(T_{Y / X}(-e)\right)$ contains the image of $\Lambda_{Y}$ under the injective homomorphism $c_{g}$. Since, by Step $1, R^{2 c} i^{\dagger} \Lambda_{X}(c)$ is isomorphic to $\Lambda_{Y}$, the required statement follows.

In the situation of Theorem 2.1, it implies the same corollaries as [Ber2], 7.4.67.4.8. In $\S 3$, the following corollary will be used.

2.3 Corollary: Suppose that $\mathcal{S}$ is a scheme of locally finite type over $\operatorname{Spec}(\mathcal{A})$, where $\mathcal{A}$ is a $k$-affinoid algebra, $(\mathcal{Y}, \mathcal{X})$ is a smooth $\mathcal{S}$-pair, $j$ is the open immersion $\mathcal{U}=\mathcal{X} \backslash \mathcal{Y} \hookrightarrow \mathcal{X}$, and $\mathcal{F}$ is an abelian sheaf on $\mathcal{X}$ which is locally isomorphic to a sheaf of the form $f^{*} \mathcal{G}$, where $\mathcal{G}$ is an étale $\Lambda_{\mathcal{S}}$-module. Then for any $q \geq 0$ there is a canonical isomorphism

$$
\left(R^{q} j_{*}\left(\left.\mathcal{F}\right|_{\mathcal{U}}\right)\right)^{\mathrm{an}} \stackrel{\sim}{\rightarrow} R^{q} j_{*}^{\text {an }}\left(\left.\mathcal{F}^{\mathrm{an}}\right|_{\mathcal{U}^{\mathrm{an}}}\right) .
$$

Proof: Using Corollary 5.2.7 from [Ber2] and its analog for schemes, it suffices to verify that $\left(R^{q} i^{!} \mathcal{F}\right)^{\text {an }} \stackrel{\sim}{\rightarrow} R^{q} i^{\text {an! }}\left(\mathcal{F}^{\text {an }}\right)$. But the latter follows from Theorem 2.1, its analog for schemes and Proposition 1.8(d).

\section{The Comparison Theorem}

3.1 Theorem: Let $\varphi: \mathcal{Y} \rightarrow \mathcal{X}$ be a morphism of finite type between schemes of locally finite type over $k$, and let $\mathcal{F}$ be a constructible abelian sheaf on $\mathcal{Y}$ with torsion orders prime to $\operatorname{char}(k)$. Then for any $q \geq 0$ there is a canonical isomorphism

$$
\left(R^{q} \varphi_{*} \mathcal{F}\right)^{\mathrm{an}} \stackrel{\sim}{\rightarrow} R^{q} \varphi_{*}^{\text {an }} \mathcal{F}^{\text {an }} .
$$


Proof: If the torsion orders of $\mathcal{F}$ are prime to $\operatorname{char}(\tilde{k})$, the theorem is proved in [Ber2], 7.5.3. We assume therefore that $\operatorname{char}(k)=0$. We may assume that $\mathcal{X}$ and $\mathcal{Y}$ are of finite type, reduced and separated and that $\mathcal{F}$ is an étale $\Lambda_{\mathcal{Y}}$-module for some $n \geq 1$, where $\Lambda=\mathbb{Z} / n \mathbb{Z}$. The theorem is proved by induction on $\operatorname{dim}(\mathcal{Y})$. It is evidently true when $\operatorname{dim}(\mathcal{Y})=0$. Assume that it is true when $\operatorname{dim}(\mathcal{Y}) \leq d-1$, where $d \geq 1$, and prove it when $\operatorname{dim}(\mathcal{Y})=d$.

STEP 1: The theorem is true if $\mathcal{X}$ is smooth, $\varphi$ is an open immersion, and $\mathcal{F}$ is constant.

We may assume that $\mathcal{Y}$ is everywhere dense in $\mathcal{X}$ and $\mathcal{F}=\Lambda_{\mathcal{Y}}$. From GAGA ([Ber1], 3.4.4) it follows that $\mathcal{Y}^{\text {an }}$ is everywhere dense in $\mathcal{X}^{\text {an }}$.

CASE $q=0: \quad$ By [SGA4], Exp. XVI, 3.2, one has $\Lambda_{\mathcal{X}} \stackrel{\sim}{\rightarrow} \varphi_{*} \Lambda_{\mathcal{Y}}$. The isomorphism $\Lambda_{\mathcal{X}^{\mathrm{an}}} \stackrel{\sim}{\rightarrow} \varphi_{*}^{\text {an }} \Lambda_{\mathcal{y}^{\mathrm{an}}}$ follows from the fact that the complement of a closed $k$-analytic subspace in a connected normal $k$-analytic space is connected. This fact follows from the Riemann Extension Theorem proved by Lütkebohmert ([Lu1], see also [Ber1], 3.3.15).

CASE $q \geq 1$ : We define an integer $l(\mathcal{Y}, \mathcal{X})$ as the length $m$ of the sequence of open subschemes $\mathcal{Y}_{0}=\mathcal{Y} \subset \mathcal{Y}_{1} \subset \cdots \subset \mathcal{Y}_{m}=\mathcal{X}$ such that $\mathcal{Y}_{i+1} \backslash \mathcal{Y}_{i}$ is the set of regular points of the reduced closed subscheme $\mathcal{X} \backslash \mathcal{Y}_{i}$. By Corollary 2.3, Step 1 is true if $l(\mathcal{Y}, \mathcal{X}) \leq 1$. Assume that it is true when $l(\mathcal{Y}, \mathcal{X}) \leq m-1$, where $m \geq 2$, and prove it when $l(\mathcal{Y}, \mathcal{X})=m$. We set $\mathcal{Z}=\mathcal{Y}_{1}$ (in the above sequence) and denote by $\mu$ (resp. $\nu$ ) the open immersion $\mathcal{Y} \hookrightarrow \mathcal{Z}$ (resp. $\mathcal{Z} \hookrightarrow \mathcal{X}$ ). Consider the morphism of spectral sequences

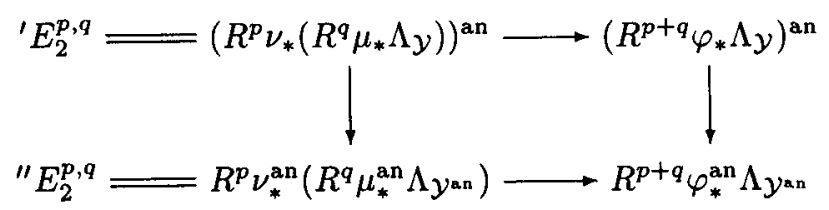

One has $\Lambda_{\mathcal{Z}} \stackrel{\sim}{\rightarrow} \mu_{*} \Lambda_{\mathcal{Y}}$ and $\Lambda_{\mathcal{Z}^{\text {an }}} \stackrel{\sim}{\rightarrow} \mu_{*}^{\text {an }} \Lambda_{\mathcal{Y}^{\text {an }}}$. Since $l(\mathcal{Z}, \mathcal{X})=m-1$ then, by induction, ${ }^{\prime} E_{2}^{p, 0} \stackrel{\sim}{\rightarrow} E_{2}^{p, 0}$ for all $p \geq 0$. Furthermore, $\mathcal{Z}^{\prime}:=\mathcal{Z} \backslash \mathcal{Y}$ is open everywhere dense in the reduced closed subscheme $\mathcal{X}^{\prime}:=\mathcal{X} \backslash \mathcal{Y}$. The sheaves $R^{q} \mu_{*} \Lambda_{\mathcal{Y}}$ (resp. $\left.R^{q} \mu_{*}^{\text {an }} \Lambda_{\mathcal{Y}_{\text {an }}}\right)$ for $q \geq 1$ are concentrated on $\mathcal{Z}^{\prime}\left(\right.$ resp. $\mathcal{Z}^{\text {an }}$ ). Since $\operatorname{dim}\left(\mathcal{Z}^{\prime}\right)<d$ then, by induction, ${ }^{\prime} E_{2}^{p, q} \simeq \simeq^{\prime \prime} E_{2}^{p, q}$ for all $p \geq 0$ and $q \geq 1$. Step 1 follows.

STEP 2: The theorem is true if $\varphi$ is an open immersion and $\mathcal{F}$ is constant. 
Let $f: \mathcal{X}^{\prime} \rightarrow \mathcal{X}$ be a resolution of singularities of $\mathcal{X}$, i.e., a proper surjective birational morphism with smooth $\mathcal{X}^{\prime}$. Then there is a commutative diagram with cartesian squares

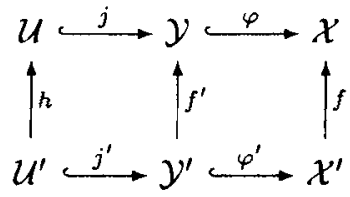

where $\mathcal{U}$ is an open everywhere dense subset of $\mathcal{Y}$ and $h$ is an isomorphism. By Step 1, the theorem is true for the pair $\left(\varphi^{\prime}, \Lambda_{y^{\prime}}\right)$. From the Comparison Theorem for cohomology with compact support ([Ber2], 7.1.4) it follows that the theorem is true for $\left(f \varphi^{\prime}, \Lambda_{\mathcal{Y}^{\prime}}\right)$. Let $i$ (resp. $\left.i^{\prime}\right)$ denote the closed immersion $\mathcal{Z}:=\mathcal{Y} \backslash \mathcal{U} \rightarrow \mathcal{Y}$ (resp. $\mathcal{Z}^{\prime}:=\mathcal{Y}^{\prime} \backslash \mathcal{U}^{\prime} \rightarrow \mathcal{Y}^{\prime}$ ). Since $\operatorname{dim}\left(\mathcal{Z}^{\prime}\right)<d$ then, by induction, the theorem is true for $\left(f \varphi^{\prime}, i^{\prime *} \Lambda_{\mathcal{Z}^{\prime}}\right)$. From the exact sequence $0 \rightarrow j_{!}^{\prime} \Lambda_{\mathcal{U}^{\prime}} \rightarrow \Lambda_{\mathcal{Y}^{\prime}} \rightarrow i^{\prime *} \Lambda_{\mathcal{Z}^{\prime}} \rightarrow 0$ it follows that the theorem is true for $\left(\varphi f^{\prime}, j_{!}^{\prime} \Lambda_{\mathcal{U}^{\prime}}\right)$. Furthermore, by the Proper Base Change Theorems for schemes and analytic spaces ([Ber2], 5.3.1), one has $R^{q} f_{*}^{\prime}\left(j_{!}^{\prime} \Lambda_{\mathcal{U}^{\prime}}\right)=0$ and $R^{q} f_{*}^{\prime \text { an }}\left(j_{!}^{\text {an }} \Lambda_{\mathcal{U}^{\prime \text { an }}}\right)=0$ for all $q \geq 1$. Since $f_{*}^{\prime}\left(j_{!}^{\prime} \Lambda_{\mathcal{U}^{\prime}}\right)=j_{!} \Lambda_{\mathcal{U}}$ and $f_{*}^{\prime \text { an }}\left(j_{!}^{\prime \text { an }} \Lambda_{\mathcal{U}^{\prime \text { an }}}\right)=j_{!}^{\text {an }} \Lambda_{\mathcal{U}^{\text {an }}}$, it follows that $R^{q} \varphi_{*}\left(j ! \Lambda_{\mathcal{U}}\right) \stackrel{\sim}{\rightarrow} R^{q}\left(\varphi f^{\prime}\right)_{*}\left(j_{!}^{\prime} \Lambda_{\mathcal{U}^{\prime}}\right)$ and $R^{q} \varphi_{*}^{\text {an }}\left(j_{!}^{\text {an }} \Lambda_{\mathcal{U}^{\text {an }}}\right) \stackrel{\sim}{\rightarrow} R^{q}\left(\varphi f^{\prime}\right)_{*}^{\text {an }}\left(j_{!}^{\text {an }} \Lambda_{\mathcal{U}^{\text {'an }}}\right)$, and therefore the theorem is true for $\left(\varphi, j ! \Lambda_{\mathcal{U}}\right)$. Finally, since $\operatorname{dim}(\mathcal{Z})<d$ then, by induction, the theorem is true for $\left(\varphi, i^{*} \Lambda_{\mathcal{Z}}\right)$. From the exact sequence $0 \rightarrow j_{!} \Lambda_{\mathcal{U}} \rightarrow \Lambda_{\mathcal{Y}} \rightarrow i^{*} \Lambda_{\mathcal{Z}} \rightarrow 0$ it follows that the theorem is true for $\left(\varphi, \Lambda_{\mathcal{Y}}\right)$.

STEP 3: The theorem is true if $\mathcal{F}$ is constant.

We may assume that $\mathcal{X}$ and $\mathcal{Y}$ are affine. Then we can represent the morphism $\varphi$ as a composition of an open immersion $j: \mathcal{Y} \hookrightarrow \overline{\mathcal{Y}}$ with a proper morphism $\bar{\varphi}: \overline{\mathcal{Y}} \rightarrow \mathcal{Y}$. By Step 2, the theorem is true for $\left(j, \Lambda_{\mathcal{Y}}\right)$ and, by the Comparison Theorem for cohomology with compact support, the theorem is true for $\left(\bar{\varphi}, R^{q} j_{*} \Lambda_{\mathcal{U}}\right)$. It follows that the theorem is true for $\left(\varphi, \Lambda_{\mathcal{Y}}\right)$.

STEP 4: The theorem is true in the general case.

We can embed any constructible sheaf $\mathcal{F}$ in a finite direct sum of sheaves of the form $f_{*} \Lambda_{\mathcal{Y}^{\prime}}$, where $f: \mathcal{Y}^{\prime} \rightarrow \mathcal{Y}$ is a finite morphism. By Step 4 , the theorem is true for $\left(\varphi f, \Lambda_{\mathcal{Y}^{\prime}}\right)$. It follows that the theorem is true for $(\varphi, \mathcal{F})$.

3.2 Corollary: Let $\mathcal{X}$ be a scheme of locally finite type over $k$, and let $\mathcal{F}$ be a constructible abelian sheaf on $\mathcal{X}$ with torsion orders prime to char $(k)$. Then for any $q \geq 0$ there is a canonical isomorphism $H^{q}(\mathcal{X}, \mathcal{F}) \stackrel{\sim}{\rightarrow} H^{q}\left(\mathcal{X}^{\text {an }}, \mathcal{F}^{\text {an }}\right)$. 
3.3 Corollary: Let $\varphi: \mathcal{Y} \rightarrow \mathcal{X}$ be a compactifiable morphism between schemes of locally finite type over $k$, and let $\mathcal{F} \in D_{c}^{b}(\mathcal{X}, \Lambda)$ with $n$ prime to $\operatorname{char}(k)$. Assume that either $n$ is prime to $\operatorname{char}(\widetilde{k})$ or $\varphi$ is a closed immersion. Then there is a canonical isomorphism

$$
(R \varphi \cdot \mathcal{F})^{\text {an }} \stackrel{\sim}{\rightarrow} R \varphi^{\text {an! }} \mathcal{F}^{\text {an }} .
$$

Proof: Suppose first that $n$ is prime to $\operatorname{char}(\tilde{k})$. Since the statement is local with respect to $\mathcal{Y}$, we may assume that $\varphi$ is the composition $\mathcal{Y} \stackrel{i}{\rightarrow} \mathcal{X}^{\prime} \stackrel{\leftrightarrow}{\rightarrow} \mathcal{X}$, where $i$ is a closed immersion and $\psi$ is smooth. By Poincaré Duality for schemes and for analytic spaces ([Ber2], 7.3.1), the statement is true for $\psi$. Thus, in both cases we may assume that $\varphi=i$ is a closed immersion. Let $j$ be the open immersion $\mathcal{X} \backslash \mathcal{Y} \hookrightarrow \mathcal{X}$. Then there is a morphism of exact triangles

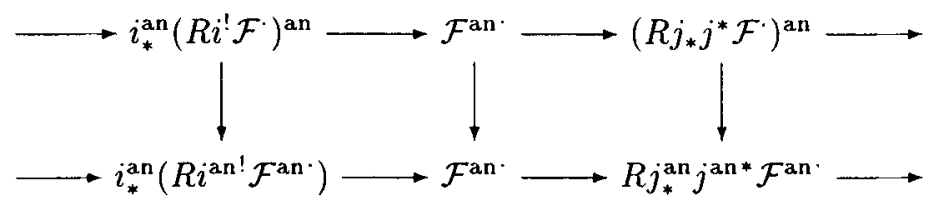

The third vertical arrow is an isomorphism, by Theorem 3.1. The statement follows.

3.4 Corollary: Let $\mathcal{X}$ be a scheme of locally finite type over $k$. Then for all $\mathcal{F}^{\cdot} \in D_{c}^{-}(\mathcal{X}, \Lambda)$ and $\mathcal{G} \in D_{c}^{+}(\mathcal{X}, \Lambda)$ with $n$ prime to char $(k)$ there is a canonical isomorphism

$$
(\underline{\mathcal{H} o m}(\mathcal{F}, \mathcal{G}))^{\text {an }} \stackrel{\sim}{\rightarrow} \underline{\mathcal{H} o m}\left(\mathcal{F}^{\text {an }}, \mathcal{G}^{\text {an }} \cdot\right)
$$

In particular, the canonical functor $D_{c}^{b}(\mathcal{X}, \Lambda) \rightarrow D^{b}\left(\mathcal{X}^{\text {an }}, \Lambda\right)$ is fully faithful.

Proof: It suffices to verify the statement when $\mathcal{F}=\mathcal{F}$ is a constructible sheaf and $\mathcal{X}$ is of finite type, separated and connected. If $\mathcal{F}$ is constant, the statement follows from Corollary 3.2. If $\mathcal{F}$ is locally constant, then there is a finite étale morphism $\varphi: \mathcal{X}^{\prime} \rightarrow \mathcal{X}$ such that $\mathcal{F}^{\prime}=\left.\mathcal{F}\right|_{\mathcal{X}^{\prime}}$ is constant. Since $\mathcal{F}$ is embedded in $\varphi_{*} \mathcal{F}^{\prime}$, the statement is easily reduced to the case of $\mathcal{F}^{\prime}$ on $\mathcal{X}^{\prime}$. In the general case, we can find an open everywhere dense subset $\mathcal{U} \subset \mathcal{X}$ such that $\left.\mathcal{F}\right|_{\mathcal{U}}$ is locally constant. Let $j$ (resp. $i$ ) be the open (resp. closed) immersion $\mathcal{U} \hookrightarrow$ $\mathcal{X}$ (resp. $\mathcal{X} \backslash \mathcal{U} \rightarrow \mathcal{X}$ ). Consider the exact sequence $0 \rightarrow j !\left(\left.\mathcal{F}\right|_{\mathcal{U}}\right) \rightarrow \mathcal{F} \rightarrow$ $i_{*} i^{*} \mathcal{F} \rightarrow 0$. Since $\underline{\operatorname{Hom}}\left(j_{1}\left(\left.\mathcal{F}\right|_{\mathcal{U}}\right), \mathcal{G}\right) \sim \underline{\sim} \underline{\operatorname{Hom}}\left(\left.\mathcal{F}\right|_{\mathcal{U}},\left.\mathcal{G}\right|_{\mathcal{U}}\right)$, then the statement is true for the sheaf $j_{!}\left(\left.\mathcal{F}\right|_{\mathcal{U}}\right)$. Furthermore, since $\underline{\operatorname{Hom}}\left(i_{*} i^{*} \mathcal{F}, \mathcal{G}\right) \stackrel{\sim}{\operatorname{Hom}}\left(i^{*} \mathcal{F}, R i^{\prime} \mathcal{G}\right)$ and 
$\operatorname{dim}(\mathcal{X} \backslash \mathcal{U})<\operatorname{dim}(\mathcal{X})$, then, by induction and Corollary 3.3 , the statement is true for $i_{*} i^{*} \mathcal{F}$. It follows that it is true for $\mathcal{F}$.

3.5. Remark: Corollary 3.3 is not true if $n$ is a power of $p=\operatorname{char}(\widetilde{k})>0$, $\operatorname{char}(k)=0$ and $\varphi$ is not a closed immersion. Indeed, assume that $k$ is algebraically closed. Then $\Lambda_{P^{1}}(1)[2] \stackrel{\sim}{\rightarrow} T_{P^{1}}$, where $P^{1}$ is the algebraic projective line. But the dualizing complex $T_{\mathbb{P} 1}$ is more complicated. For example, if $D$ is an open disc in $\mathbb{P}^{1}$, then the group $H_{c}^{2}\left(D, \mu_{p}\right)$ is very big (see [Ber2], 6.2.10), and therefore $\left.T_{\text {P1 }}\right|_{D} \underset{\rightarrow}{\sim} T_{D}$ is not isomorphic to $\Lambda_{D}(1)[2]$. It would be interesting to understand the structure of the dualizing complexes in this situation.

\section{References}

[Ber1] V. B. Berkovich, Spectral theory and analytic geometry over non-Archimedean fields, Mathematical Surveys and Monographs, vol. 33, American Mathematical Society, Providence, R.I., 1990.

[Ber2] V. B. Berkovich, Étale cohomology for non-Archimedean analytic spaces, Publications Mathématiques de l'IHES 78 (1993), 5-161.

[Ber3] V. B. Berkovich, Vanishing cycles for formal schemes, Inventiones mathematicae 115 (1994), 539-571.

[God] R. Godement, Topologie Algébrique et Théorie des Faisceux, Hermann, Paris, 1958.

[Lu1] W. Lütkebohmert, Der Satz von Remmert-Stein in der nichtarchimedischen Funktionentheorie, Mathematische Zeitschrift 139 (1974), 69-84.

[Lu2] W. Lütkebohmert, Riemann's existence problem for a $p$-adic field, Inventiones mathematicae 111 (1993), 309-330.

[SGA4] M. Artin, A. Grothendieck and J.-L. Verdier, Théorie des Topos et Cohomologie Étale des Schémas, Lecture Notes in Math. 269, 270, 305, Springer, Berlin-Heidelberg-New York, 1972-1973. 